\title{
Preparation of Bio-Functionalized Surfaces with an Electrochemically Active Silane Presenting Concealed Aldehyde ${ }^{\dagger}$
}

\author{
Hyejin Park, Al-Monsur Jiaul Haque, and Kyuwon Kim* \\ Department of Chemistry, University of Incheon, Incheon 406-772, Korea. ${ }^{*}$ E-mail: kyuwon_kim@incheon.ac.kr \\ Received September 14, 2010, Accepted October 6, 2010
}

Key Words: Electroactive silane, Protein immobilization, Aldehyde, 1.3-Dithiane, Indium-tin-oxide

A chemical modification of surfaces with organic anchor molecules has been intensively investigated in biosensors and bioelectronics application of glass and metal oxide substrates. Organosilane combined with a reactive end group such as a primary amine- or thiol-group has been widely employed for the modification where silane part is attached on the hydroxylated surface through silanization process. In spite of the wide usage of the silanes, the silanized surfaces need a further functionalization for the immobilization of biomolecules or their ligands by chemical coupling reactions with a biomolecule-reactive group such as $N$-hydroxysuccinimide (NHS). ${ }^{1}$

Potential alternative is to use of an aldehyde group-terminated silane because it can be directly coupled to amine-terminated molecules without the further activation steps. The limitation of the use is a lack of stability of aldehyde group during synthesis, storage, and attachment process. ${ }^{2,3}$ To overcome the drawback, it is desirable that the group is protected before use and even during the surface modification process. After the modification the group is on-demand deprotected by external stimuli. ${ }^{4}$ We previously reported aryldiazonium cation coupled with $1,3-$ dithiane that is an electroactive group that can afford to produce aldehyde functionality through an electrochemical oxidation. ${ }^{5}$ In this study, we report a new silane combined with 1,3-dithiane (SID) and its use in the preparation of bio-surfaces through ondemand formation of aldehyde group. We employed indiumtin-oxide (ITO) film on glass as an electrode surface for the silanization process because it has prominent characteristics for biosensors based on the electrochemical principle, such as a wide electrochemical working window and high optical transparency. ${ }^{6}$

Scheme 1 displays an attachment of SID on ITO surface through silanization process, an electrochemically oxidative deprotection of aldehyde group, and an immobilization of bioreactive group onto the resulting surface. Under the applied anodic potential, 1,3-dithiane can be oxidized and deprotected to lead aldehyde and disulfide group as reported in the previous papers. ${ }^{5,7}$ The electrochemical oxidative deprotection was examined with cyclic voltammetry (CV) and X-ray photoelectron spectroscopy (XPS). Figure 1A shows CVs obtained with the surface after the silanization for $2 \mathrm{~h}$. While an irreversible anodic peak was observed at about $1.2 \mathrm{~V}$ on the first scan, it disappeared on the second scan, which is similar to the behaviors of $\mathrm{CVs}$ at the previous reports for the electrochemical depro-

${ }^{\dagger}$ This paper is dedicated to Professor Hasuck Kim for his outstanding contribution to electrochemistry and analytical chemistry.

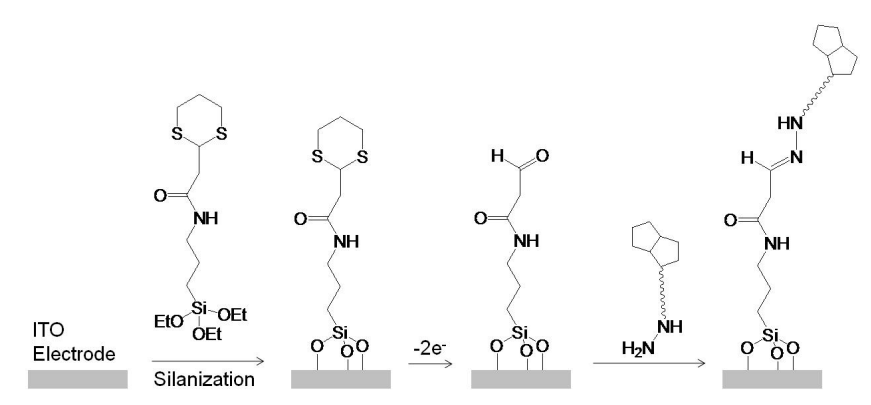

Scheme 1. A proposed scheme for an attachment of SID on ITO surfaces through silanization, an electrochemical oxidation for the deprotection of aldehyde, and a covalent immobilization of biotin hydrazide onto the aldehyde group.

(A)

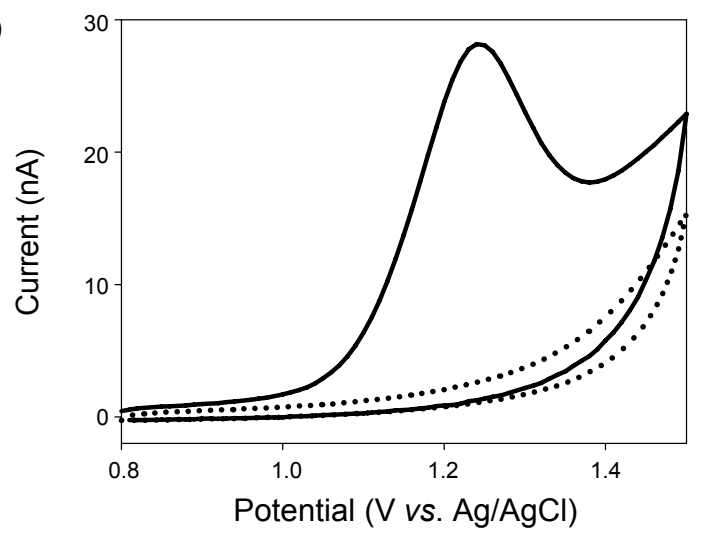

(B)

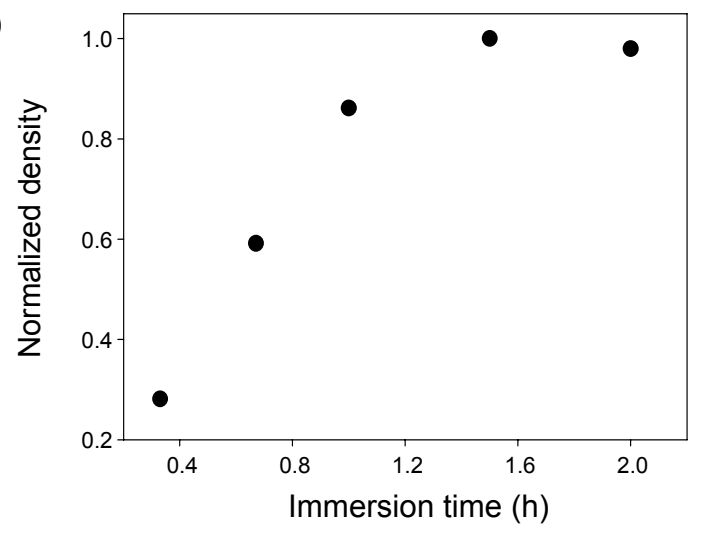

Figure 1. (A) CVs for the electrochemical oxidations of SID modified ITO surfaces in an acetonitrile solution with $0.1 \mathrm{M}$ TBAP (all scan rates $=50 \mathrm{mV} / \mathrm{sec}$ ); Solid line, first cycle; dotted line, second cycle. (B) A variance of the electrical charge required to the electrochemical oxidation as a function of immersion time for the silanization. The value is normalized. 
tection of aldehyde group. ${ }^{4,5}$ To monitor the silanization, we changed a cleaning and a hydroxylation condition of ITO surfaces, and then evaluated charge required for the electrochemical oxidation by observing $\mathrm{CV}$ as a function of immersion time of the substrate in the silane solution. Figure 1B shows normalized charge obtained from CVs. The result reveals that the charge needed for the electrochemical oxidation increases with increase in time up to $1.5 \mathrm{~h}$, then levels off with further immersion, which implies a saturation of the surface with silane within that time window. It should be noted that the observation of electrochemical parameters such as peak currents or charges provides a more simple way to predict the degree of silanization on the surface than optical methods such as ellipsometry or XPS. To the best of our knowledge, the electrochemical monitoring of silanization has never been reported. The optimized surface density of SID was estimated from the peak area of $\mathrm{CV}$ with an approximation of 2-electron process. It was found to be $3.1 \times$ $10^{-10} \mathrm{~mol} / \mathrm{cm}^{2}$ with the electrode area of $0.07 \mathrm{~cm}^{2}$, employed in this estimation without considering surface roughness. The value is slightly lower than the density of $6.3 \times 10^{-10} \mathrm{~mol} / \mathrm{cm}^{2}$, expected for the saturated monolayer with an assumption that the area occupied by a single silane molecule is $0.28 \mathrm{~nm}^{2} .8$ This result may mainly be due to incomplete electrochemical oxidation of the dithiane group tethered on the surface.

The formation of aldehyde on the oxidized surface was examined with XPS for carbon (C1s) atoms. Figure 2A compares two normalized results of $\mathrm{C}(1 \mathrm{~s})$ before and after the oxidation. Two peaks at 288.6 and $285.0 \mathrm{eV}$ due to the carbonyl atom of amide part of SID and the other carbon on the surface, respectively, were commonly observed at both cases before the oxidation. However, the ratio of the former to the latter peak increased after the oxidation, which is attributed to the relative increase of carbonyl carbon atom on the surface. ${ }^{5}$ This increase also supports the formation of aldehyde after the electrochemical deprotection because a binding energy of the carbonyl carbon in aldehyde is nearly same as the one in amide group.

To prepare bio-surfaces making use of the reactivity of aldehyde of the silane, biotin hydrazide as a ligand molecule of avidin was employed because it is well known that a reaction of hydrazide and aldehyde forms a stable product of hydrazone. The silanized ITO surface was subjected to the electrochemical deprotection, and then the treated surface was exposed to the solution of $10 \mathrm{mM}$ biotin hydrazide. A reactivity of the biotin was investigated with a N(1s) peak in XPS and an immobilization of avidin labeled with TRITC (tetramethylrhodamine isothiocyanate) on the resulting surface. In the course of examination, we observed that an acidity of the biotin solution was an important factor to maximize the reactivity and an acidic condition of $\mathrm{pH} 2.3$ gave a better result in the peak intensity, as seen in Figure 2B that displays the XPS results from two different $\mathrm{pH}$ values. The $\mathrm{pH}$ dependence is unexpected because a nucleophilicity of hydrazide toward aldehyde decreases with a $\mathrm{pH}$ decrease. The reverse reactivity might be caused by the chemical structure of the deprotected silane presenting aldehyde functionality and a very low $\mathrm{p} K_{\mathrm{a}}$ value $(\sim 3)$ for the terminal amine of the hydrazide. ${ }^{9}$ A proton on the carbon between two carbonyl groups in the structure is so acidic that it can be easily removed from the carbon, which can afford to change from keto-
(A)

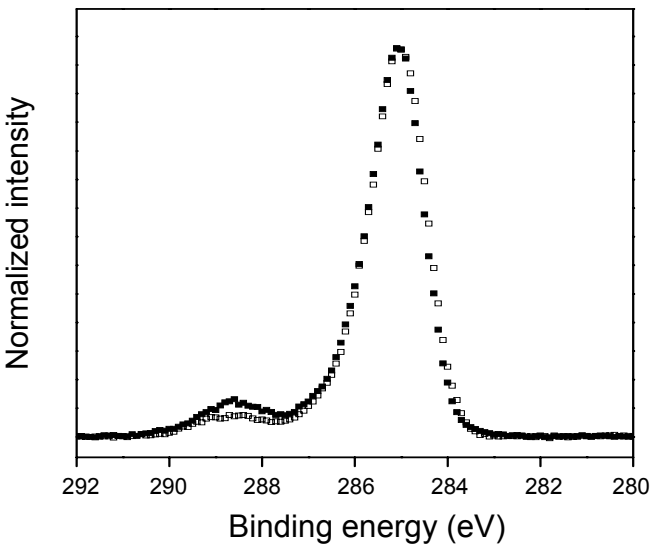

(B)

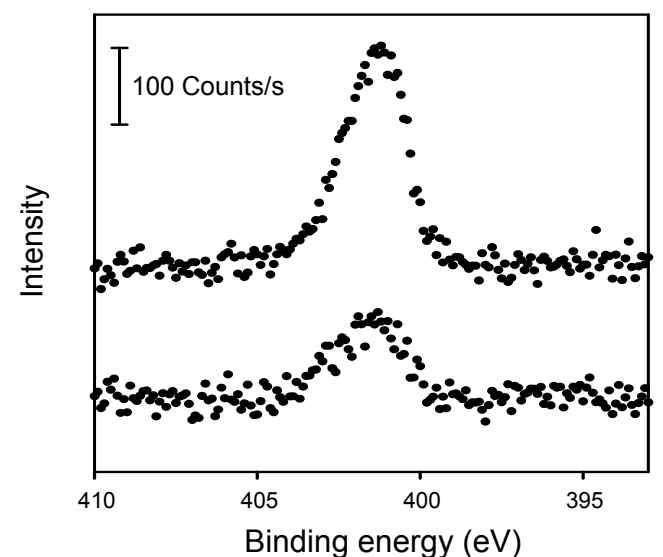

Figure 2. (A) Normalized XPS results of C(1s) for SID-modified ITO surfaces before ( $\square$ ) and after ( $\square$ ) the electrochemical oxidation. (B) XPS results of N(1s) for the aldehyde surfaces after the treatment of with biotin hydrazide in the buffer solution of $\mathrm{pH} 2.3$ (top) and 7 (bottom).

to enol-form at even neutral $\mathrm{pH}$, resulting in low reactivity toward the hydrazide. On the other hand, keto-form is dominant at lower $\mathrm{pH}$ and a loss of the nucleophilicity of the hydrazide is not serious owing to its low $\mathrm{p} K_{\mathrm{a}}$ characteristic. A test of the reaction using a biotin derivatized with a primary amine instead of hydrazide gave low signal regardless of $\mathrm{pH}$ values even after the reduction of the imine product by treatment of $\mathrm{NaBH}_{3} \mathrm{CN}$. The reverse reactivity of the silanized surface needs still more studies because it is unusual and $\mathrm{pH}$ dependent behavior is a very important part of the surface chemical reaction. ${ }^{10}$

We prepared a bio-surface based on the electrochemical addressing. A band type ITO microelectrodes patterned glass was used as a platform, which is prepared and treated as described in the previous report. ${ }^{11}$ Avidin as a model protein has been frequently used to demonstrate a feasibility of a new immobilization method because the biospecific interaction between biotin and avidin (association constants, $K_{\mathrm{a}} \sim 10^{15} \mathrm{M}^{-1}$ in solution) is very strong. ${ }^{12}$ After the silanization of the whole substrate including four electrodes, as shown in the top of Figure 3, by exposure to the solution of $1 \mathrm{mM}$ SID, only one electrode among four ones was addressed with an electrochemical oxidation potential of $1.1 \mathrm{~V}$ for the deprotection of aldehyde. The resulting whole substrate was exposed to the $\mathrm{pH}$-adjusted buffered solution of $10 \mathrm{mM}$ biotin hydrazide. After washing, the surface was 


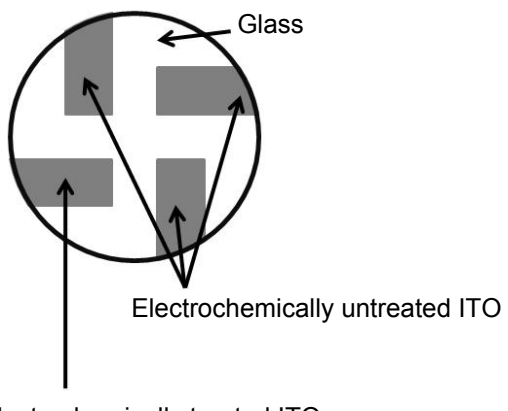

Electrochemically treated ITO
(A)

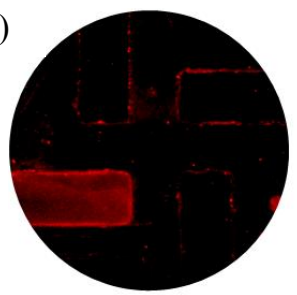

(C)

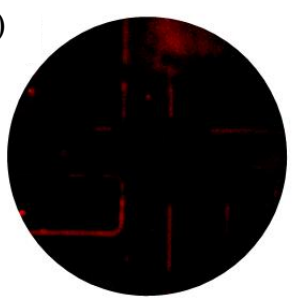

(B)

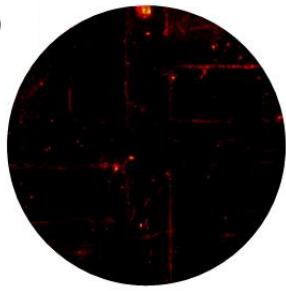

(D)

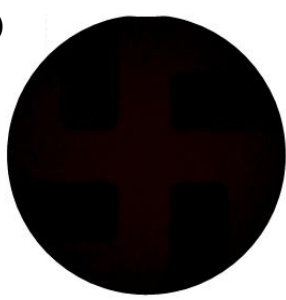

Figure 3. Fluorescence microscopic images after the spatially selective immobilization of TRITC-labeled avidin on the microelectrode array surfaces prepared from the biotin attachment in the solution $\mathrm{pH}$ (A) 2.3, (B) 4.0, (C) 7.0, and (D) 10.0 for after the electrochemical deprotection of only one electrode of each array surface. Width of each electrode $=150 \mu \mathrm{m}$.

disclosed to the solution of $10 \mu \mathrm{g} / \mathrm{mL}$ TRITC-labeled avidin. Figure 3 shows that fluorescence microscopic images of avidin surfaces obtained from four different $\mathrm{pH}$ conditions of the attachment step of biotin hydrazide. While a clear red color due to TRITC is exclusively seen on the electrode subjected to $\mathrm{pH} 2.3$, there is no distinctive color on the electrode for higher $\mathrm{pHs}$. The better immobilization of avidin indicates the higher surface density of biotin. This is consistent to the $\mathrm{pH}$-dependent behavior of biotin density reveled with XPS study for biotin surface, as shown in Figure 2. From avidin immobilization study together with the XPS results, we presume the absolute density of the attached biotin is not large even under the acidic $\mathrm{pH}$, because it is well known that the largest yield of avidin immobilization is found at one tenth of a full monolayer density of biotin. ${ }^{13}$ The exclusive pattern result from highly acidic $\mathrm{pH}$ indicates that the attachment of biotin hydrazide is negligible on the other electrodes and glass surfaces presenting the protected aldehyde, which supports a high spatial selectivity of our immobilization method based on electrochemical addressing.

In conclusion, we have demonstrated the use of SID as a new electroactive silane, for the preparation of bio-surfaces based on the spatially selective manner as well as $\mathrm{pH}$ dependent attachment of bio-ligand. The SID is one of the electroactive anchor molecules utilized in on-demand initiation of surface reactions, and in preparation of surfaces with multiplex probes to detect multiple targets through the electrochemical addressing of electrodes. Together with this usefulness, SID could be employed for the study requiring the highly selective attachment of materials with minimizing the interference of a simple primary amine.

\section{Experimental Section}

Biotin hydrazide and TRITC-labeled avidin were purchased from Sigma. Phosphate Buffer Saline (PBS) solution consisted of $0.01 \mathrm{M}$ phosphate and $0.15 \mathrm{M} \mathrm{NaCl}$. PBST is composed of PBS and $0.05 \%(w / w)$ tween-20. Citric acid buffer (CAB) solution consisted of $0.1 \mathrm{M}$ citric acid and $0.1 \mathrm{M}$ sodium citrate. Detailed synthetic procedure of SID is described in Supporting information.

ITO microelectrode was immersed in $1 \mathrm{mM}$ of SID solution for $2 \mathrm{~h}$, after successively cleansing with ethanol, acetone and water, immersing in piranha solution for 5 seconds, and washing with pure water. The resulting SID -modified ITO surface was subjected to the electrochemical oxidation of only one electrode in acetonitrile containing $0.1 \mathrm{M}$ tetrabutyl ammonium perchlorate (TBAP). After that, the ITO surface was immersed in water for $30 \mathrm{~min}$. This was followed by washing with pure water and dry with $\mathrm{N}_{2}$ gas. After that, the whole surface was treated with a $10 \mu \mathrm{L}$ drop of $10 \mathrm{mM}$ biotin hydrazide solution in CAB at $\mathrm{pH} 2.35$ and incubated for $30 \mathrm{~min}$ at room temperature. This was followed by washing with pure water and dry with $\mathrm{N}_{2}$ gas. Afterward, the surface was treated with a $10 \mu \mathrm{L}$ drop of $10 \mu \mathrm{g} /$ $\mathrm{mL}$ TRITC-labeled avidin solution in PBST and incubated for 30 min at room temperature. After washing with PBST and water and drying with $\mathrm{N}_{2}$ gas, the surface was subjected to the observation with fluorescent microscope (IX 71, Olympus, Tokyo, Japan).

Acknowledgments. This work was supported by University of Incheon Research Grant in 2008.

\section{References}

1. Frey, B. L.; Corn, R. M. Anal. Chem. 1996, 68, 3187.

2. Ghasemi, M.; Minier, M.; Tatoulian, M.; Arefi-Khonsari, F. Langmuir 2007, 23, 11554-11561.

3. Peelen, D.; Smith, M. L. Langmuir 2005, 21, 266.

4. Yeo, W. S.; Mrksich, M. Adv. Mater. 2004, 16, 1352-1356.

5. Haque, A.-M. J.; Kwon, S.-R.; Park, H.; Kim, T.-H.; Oh, Y.-S.; Choi, S.-Y.; Hong, J.-D.; Kim, K. Chem. Commun. 2009, 4865.

6. Manifacier, J. Thin Solid Films 1982, 90, 297.

7. Martre, M.; Mousset, G.; Rhlid, R. B.; Veschambre, H. Tetrahedron Lett. 1990, 31, 2599.

8. Mittal, K. L. Silanes and Other Coupling Agents; VSP: Utrecht, The Netherlands, 2000; Vol. 2.

9. Zalipisky, S.; Milton H. J. Polyethylene Glycol; American Chemical Society: 1997; pp 318-341.

10. Hagan, K. A.; Meier, W. L.; Ferrance, J. P.; Landers, J. P. Anal. Chem. 2009, 81, 5249.

11. Yu, D.; Kim, K. Bull. Korean Chem. Soc. 2009, 30, 955.

12. Kirley, T. L. Anal. Biochem. 1989, 10, 231.

13. Spinke, J.; Liley, M.; Schmitt, F. J.; Guder, H. J.; Angermaier, L.; Knoll, W. J. Chem. Phys. 1993, 99, 7012. 